\title{
МОДЕЛИРОВАНИЕ ЧИСЛЕННОСТИ ВЫПУСКА ИНЖЕНЕРНО-ТЕХНИЧЕСКИХ СПЕЦИАЛИСТОВ
}

\author{
Кочеткова Е.В.
}

В работе предложена модель выпуска инженерно-технических специалистов, учитываюмая динамику приема абитуриентов на инженерно-технические и другие спещиальности, а также рассмотрена возможность моделирования коэффициента смены специальности в прочессе обучения, косвенно отражающего ожидания будущего спроса на выбираемую профессию.

DOI: $10.20537 /$ mce2019econ13

Введение. Актуальной в настоящее время как для России, так и для зарубежных стран является проблема несбалансированности спроса и предложения на рынке труда инженерно-технических специалистов (ИТС) $[1,2]$. В качестве краткосрочной меры ликвидации кадрового дефицита нередко рассматривается привлечение квалифицированных специалистов из-за рубежа. Тем не менее, подчеркивается необходимость долгосрочного планирования и прогнозирования потребности в кадрах на перспективу с учетом целевых показателей экономического развития $[3,4]$.

Достаточно часто несбалансированности спроса и предложения сопутствует весьма высокая профессиональная мобильность специалистов, смена специальности и отсев в процессе обучения (dropout), являющиеся следствием и усиливающая проблему профессиональноквалификационного несоответствия требований работодателей и предложения труда (field/qualification mismatch). По различным оценкам, доля работающих не по полученной специальности или в несоответствующей квалификации в европейских странах, США, а также в России могла достигать более $40 \%$ (см. табл. 1 ).

Эти проблемы свидетельствуют о необходимости разработки моделей для анализа и прогноза динамики выпуска. При этом, учитывая высокий уровень мобильности специалистов и, особенно, получающих среднее и высшее образование, целесообразна разработка моделей, позволяющих анализировать не только выпуск, но и динамику приема абитуриентов, смены специальности и отсева в процессе обучения (см. также [5]). 
Анализ и моделирование экономических и сочиальных прочессов - МКО - 2019 Analysis and modeling of economic and social processes - MCE - 2019

Таблица 1. Оценки численности специалистов, сменивших направление во время обучения или работавших вне своей специальности или квалификации.

\begin{tabular}{|c|c|c|c|}
\hline $\begin{array}{l}\text { Вид мобильности / } \\
\text { несоответствия }\end{array}$ & Страна & $\begin{array}{l}\text { Оценка численности специалистов, } \\
\text { работавших вне своей специальности }\end{array}$ & $\begin{array}{l}\text { Ис- } \\
\text { Точ } \\
\text { ниК }\end{array}$ \\
\hline \multirow{3}{*}{$\begin{array}{l}\text { Смена направле- } \\
\text { ния в процессе } \\
\text { обучения (drop- } \\
\text { out), \% от чис- } \\
\text { ленности посту- } \\
\text { пивших на пер- } \\
\text { вый курс }\end{array}$} & \multirow{3}{*}{ США } & около $30 \%$ в 1998 г. & {$[6]$} \\
\hline & & $\begin{array}{l}30-50 \% \text { студентов инженерных спе- } \\
\text { циальностей в } 2004 \text { г. }\end{array}$ & [7] \\
\hline & & $\begin{array}{l}52 \% \text { студентов математических, } \\
40 \% \text { естественнонаучных, } \\
32 \% \text { инженерно-технических специ- } \\
\text { альностей в 2011-2012 гг. }\end{array}$ & [8] \\
\hline \multirow{10}{*}{$\begin{array}{l}\text { Несоответствие } \\
\text { работы специ- } \\
\text { альности обуче- } \\
\text { ния (field mis- } \\
\text { match) }\end{array}$} & \multirow{3}{*}{$\begin{array}{l}\text { Великобри- } \\
\text { тания }\end{array}$} & $18.6 \%$ от численности занятых в 2005 г. & [9] \\
\hline & & $46 \%$ выпускников в 2010 г. & [10] \\
\hline & & 50\% выпускников в 2014 г. & {$[11]$} \\
\hline & Испания & 6\% от численности занятых в 2005 г. & [9] \\
\hline & Нидерланды & $11.1 \%$ от численности занятых в 2005 г. & [9] \\
\hline & Япония & $24.2 \%$ от численности занятых в 2005 г. & [9] \\
\hline & Герм & $10.4 \%$ от численности занятых в 2005 г. & [9] \\
\hline & США & $\begin{array}{l}19 \% \text { от численности занятых среди } \\
\text { мужчин и } 21 \% \text { среди женщин в } 2007 \text { г. }\end{array}$ & {$[12]$} \\
\hline & Швеция & $\begin{array}{l}16 \% \text { от численности занятых среди } \\
\text { мужчин и } 10 \% \text { среди женщин в } 2008 \text { г. }\end{array}$ & {$[13]$} \\
\hline & Россия & $\begin{array}{l}\text { около 50\% выпускников вузов и около } \\
60 \% \text { выпускников ссузов инженерно- } \\
\text { технических специальностей в } 2009 \text { г. }\end{array}$ & [14] \\
\hline
\end{tabular}

Разработка модели. Исследования проблемы смены специальности в процессе обучения тесно связаны с выделением междисциплинарных аспектов - экономических, социологических, психологических [15-17]. Анализ показал, что необходимо учитывать следующие группы факторов: индивидуальные (наличие способностей и склонностей к данной профессии), социально-психологические (социализация, положительная оценка труда и проч.), социально-экономические (обычно согласующиеся с предположениями теории человеческого капитала), внешние (наличие вакансий в данной специальности, спрос со стороны других отраслей, региональная специфика), см. [18-20]. 
Поскольку большинство известных моделей численности выпуска специалистов либо не включают блок, в котором бы учитывалась смена специальности в процессе обучения, ссылаясь на то, что гипотеза о наличии взаимосвязи численности выпуска специалистов и величиной заработной платы в момент поступления абитуриентов недостаточно глубоко разработана [7], либо рассматривают долю выбывших как некоторую постоянную величину (например, в [21]), при разработке модели возникает задача выбора в качестве эндогенной переменной показателя приема или выпуска специалистов, а также моделирования функции, описывающей число специалистов, меняющих профессию в процессе обучения или покидающих обучение по другим причинам.

Приведенная в данной работе динамическая модель численности выпуска инженерно-технических специалистов с учетом динамики приема и смены специальности в процессе обучения (модель 1) является развитием предложенной ранее в работе [22] нелинейной модели для анализа взаимной динамики численности выпуска специалистов.

В настоящей работе рассматривались две укрупненные группы специалистов - учащиеся на инженерно-технических и на всех остальных специальностях. При этом выделялись группы получающих среднее (СПО) и высшее профессиональное образование (ВПО). Таким образом, модель состоит из трех блоков:

1. численность приема на инженерно-технические специальности:

$\Delta E_{1, t} / E_{1, t-1}=a_{0}+a_{1} E_{1, t-1}+a_{2} O_{l, t-1}+m_{t} / s_{t}$,

$\Delta O_{1, t} / O_{1, t-1}=a_{3}+a_{4} E_{1, t-1}+a_{5} O_{1, t-1}$,

$\Delta E_{2, t} / E_{2, t-1}=a_{6}+a_{7} E_{2, t-1}+a_{8} O_{2, t-1}+a_{9} y i_{t}$,

$\Delta O_{2, t} / O_{2, t-1}=a_{10}+a_{11} E_{2, t-1}+a_{12} s_{t}$,

2. коэффициенты смены специальности в процессе обучения:

$k_{1, t}=c_{0}+c_{1} E_{2, t}+c_{2} L i_{t}$,

$k_{2, t}=c_{3}+c_{4} W_{t}+c_{5} L i_{t}+c_{6} D$,

3. численность выпуска ИТС:

$G E_{1, t}=k_{1, t} E_{1, t}$,

$G E_{2, t}=k_{2, t} E_{2, t}$,

$G E_{t}=G E_{1, t}+G E_{2, t}$,

где $E_{1, t}, E_{2, t}$ - прием на инженерно-технические специальности СПО и ВПО соответственно; $O_{1, t}, O_{2, t}$ - прием на все остальные специальности СПО и ВПО соответственно; $m_{t}$ - темп прироста выпуска обрабаты- 
вающей промышленности; $s_{t}$ - темп прироста выпуска сектора услуг; $y i_{t}$ темп прироста выпуска промышленности и строительства; $L i_{t}-$ численность занятых в промышленности; $W_{t}$ - отношение средней начисленной заработной платы в обрабатывающих производствах и финансовой и профессиональной деятельности; $\mathrm{D}=\{1, \mathrm{t} \in\{2012,2013\} ; 0, \mathrm{t} \notin\{2012,2013\}\}$.

Источниками статистической информации служили данные Росстата [23] и сборники ВШЭ [24].

Результаты моделирования позволяют получить удовлетворительное качество аппроксимации фактическим данным: относительная среднеквадратическая ошибка для переменной численности выпуска ИТС со средним специальным образованием $\left(G E_{1, t}\right)$ составила $8 \%$, для переменной численности выпуска ИТС с высшим образованием $\left(G E_{2, t}\right)-4 \%$; для переменной численности выпуска ИТС в целом $\left(G E_{t}\right)$ также $4 \%$ (см. рис. 1-2).

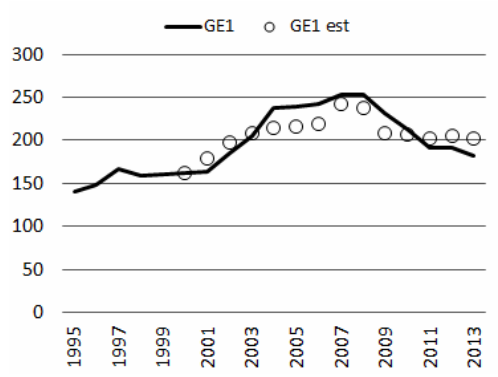

Рис.1. Фактические $\left(G E_{1, t}\right)$ и полученные в результате моделирования $\left(G E_{l, t}\right.$ est $)$ по модели 2 значения численности выпуска ИТС со средним специальным образованием.

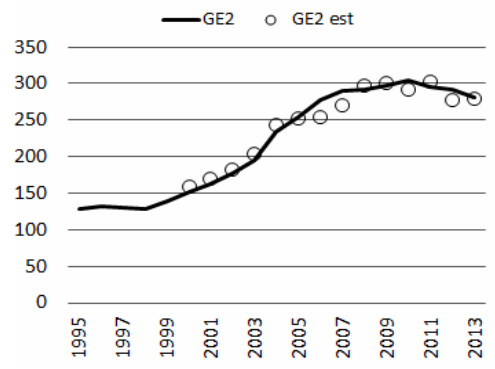

Рис.2. Фактические $\left(G E_{2, t}\right)$ и полученные в результате моделирования $\left(G E_{2, t}\right.$ est) по модели 2 значения численности выпуска ИТС с высшим образованием.

В целях анализа ожидаемого дисбаланса спроса и предложения ИТС была рассмотрена возможность включения разработанной расширенной модели в качестве блока в макроэкономическую модель. Полученная макромодель (модель 2), таким образом, состояла из 5 блоков:

1. численность выпуска инженерно-технических специалистов с учетом динамики приема и смены специальности в процессе обучения

$$
\begin{aligned}
\Delta E_{1, t} / E_{1, t-1} & =a_{0}+a_{1} E_{1, t-1}+a_{2} O_{1, t-1}+m_{t} / s_{t}, \\
\Delta O_{1, t} / O_{1, t-1} & =a_{3}+a_{4} E_{1, t-1}+a_{5} O_{1, t-1},
\end{aligned}
$$




$$
\begin{aligned}
& \Delta E_{2, t} / E_{2, t-1}=a_{6}+a_{7} E_{2, t-1}+a_{8} O_{2, t-1}+a_{9} y i_{t}, \\
& \Delta O_{2, t} / O_{2, t-1}=a_{10}+a_{11} E_{2, t-1}+a_{12} S_{t}, \\
& k_{1, t}=c_{0}+c_{1} E_{2, t}+c_{2} L i_{t}, \\
& k_{2, t}=c_{3}+c_{4} W_{t}+c_{5} L i_{t}+c_{6} D, \\
& G E_{1, t}=k_{1, t} E_{1, t}, \\
& G E_{2, t}=k_{2, t} E_{2, t}, \\
& G E_{t}=G E_{1, t}+G E_{2, t}, \\
& 2 . \text { ВДС сектора услуг } \\
& S_{t}=b_{0}+b_{1} Y_{t-1}+b_{2} S_{t-1}+b_{3} \Delta Y_{t-1}+b_{4} \Delta S_{t-1},
\end{aligned}
$$

3. производительность труда в промышленности

$\operatorname{Ln}\left(Y i_{t} / L i_{t}\right)=b_{5}+b_{6} \operatorname{Ln}\left(I i_{t} / L i_{t}\right)+b_{7} \operatorname{Ln}\left(E T_{t} / L i_{t}\right)$,

4. численность занятых инженерно-технических специалистов $E T_{t}=b_{8} E T_{t-1}+b_{9} G E_{\mathrm{t}}$,

5. производительность труда в экономике

$$
\operatorname{Ln}\left(Y_{t} / L_{t}\right)=b_{10}+b_{11} \operatorname{Ln}\left(F_{t} / L_{t}\right)+b_{12} \operatorname{Ln}\left(Y i_{t} / L_{t}\right) \text {, }
$$

где $Y_{t}-$ ВВП; $F_{t}$ - основные фонды; $L_{t}$ - численность занятых; $E T_{t}-$ численность ИТС, $S_{t-1}$ - ВДС сектора услуг; $Y i_{t}-$ выпуск промышленных производств, $I i_{t}$ - инвестиции в основные фонды промышленности, $L i_{t}$ - численность занятых в промышленности. Оценки параметров уравнений (1)-(13) модели 2 представлены в табл. 2.

Предложенная макромодель позволяет получить достаточно точные оценки (относительная среднеквадратическая ошибка для переменной численности выпуска ИТС $\left(G E_{t}\right)$ для предложенной модели с учетом динамики приема составила 5\%), однако все же несколько уступающие по точности ранее разработанной модели (подробнее см. [25], относительная среднеквадратическая ошибка для переменной численности выпуска ИТС $\left(G E_{t}\right)$ составила $2 \%$ ). Таким образом, стоит рассматривать возможность ее использования при дополнительной доработке в некоторых специальных случаях.

Выводы. В работе предложен подход к построению динамической модели выпуска ИТС, позволяющей учесть влияние социальноэкономических аспектов выбора специальности абитуриентами, а также делает возможным отражение факторов, влияющих на возможность ухода обучающегося из учебного заведения или из специальности. В дальнейшем, по нашему мнению, при разработке макромоделей для анализа потребности в ИТС необходимо учитывать также проблемы профессиональной мобильности выпускников и работающих инженеров. 
Анализ и моделирование экономических и сочиальных прочессов - МКО - 2019 Analysis and modeling of economic and social processes - MCE - 2019

Таблица 2. Оценки параметров модели 2, уравнения (1)-(13).

\begin{tabular}{|c|c|c|c|}
\hline $\begin{array}{l}\text { Зависимая пере- } \\
\text { менная }\end{array}$ & Фактор & $\begin{array}{c}\text { Оценка параметра } \\
\text { (t-статистика) }\end{array}$ & $\mathrm{R}^{2}$ \\
\hline \multirow[t]{4}{*}{$\Delta E_{1, t} / E_{1, t-1}$} & const & $8.399(0.854)$ & \multirow[t]{4}{*}{0.53} \\
\hline & $E_{1, t-1}$ & $-0.063(-2.478)$ & \\
\hline & $O_{1, t-1}$ & $0.018(1.193)$ & \\
\hline & $m_{t} / s_{t}$ & $1.742(2.588)$ & \\
\hline \multirow{3}{*}{$\Delta O_{1, t} / O_{1, t-1}$} & const & $13.754(1.436)$ & \multirow[t]{3}{*}{0.43} \\
\hline & $E_{1, t-1}$ & $-0.081(-3.270)$ & \\
\hline & $O_{1, t-1-1}$ & $0.017(1.120)$ & \\
\hline \multirow[t]{4}{*}{$\Delta E_{2, t} / E_{2, t-1}$} & const & $27.889(4.812)$ & \multirow[t]{4}{*}{0.63} \\
\hline & $E_{2, t-1}$ & $-0.135(-3.260)$ & \\
\hline & $O_{2, t-1}$ & $0.018(1.716)$ & \\
\hline & $y i_{t}$ & $0.400(2.051)$ & \\
\hline \multirow[t]{3}{*}{$\Delta O_{2, t} / O_{2, t-1}$} & const & $44.163(5.834)$ & \multirow[t]{3}{*}{0.69} \\
\hline & $E_{2, t-1}$ & $-0.136(-5.657)$ & \\
\hline & $s_{t}$ & $0.853(2.658)$ & \\
\hline \multirow{3}{*}{$k_{l, t}$} & const & $-0.542(-2.503)$ & \multirow[t]{3}{*}{0.76} \\
\hline & $E_{2, t}$ & $0.001(4.094)$ & \\
\hline & $L i_{t}$ & $0.054(5.183)$ & \\
\hline \multirow[t]{4}{*}{$k_{2, t}$} & const & $-0.801(-1.557)$ & \multirow[t]{4}{*}{0.67} \\
\hline & $L i_{t}$ & $0.056(2.787)$ & \\
\hline & $W_{t}$ & $2.208(3.053)$ & \\
\hline & $D$ & $-0.124(-3.370)$ & \\
\hline \multirow[t]{5}{*}{$S_{t}$} & const & $-1748.622(-3.416)$ & \multirow[t]{5}{*}{0.99} \\
\hline & $Y_{t-1}$ & $0.465(7.949)$ & \\
\hline & $S_{t-1}$ & $0.322(3.948)$ & \\
\hline & $\Delta S_{t-1}$ & $0.432(2.172)$ & \\
\hline & $\Delta Y_{t-1}$ & $-0.260(-2.039)$ & \\
\hline \multirow[t]{2}{*}{$E T_{t}$} & $E T_{t-1}$ & $0.975(14.440)$ & \multirow[t]{2}{*}{0.99} \\
\hline & $G E_{\mathrm{t}}$ & $0.606(0.842)$ & \\
\hline \multirow[t]{3}{*}{$\operatorname{Ln}\left(Y i_{t} / L i_{t}\right)$} & const & $4.406(6.130)$ & \multirow[t]{3}{*}{0.95} \\
\hline & $\operatorname{Ln}\left(I i_{t} / L i_{t}\right)$ & $0.642(6.024)$ & \\
\hline & $\operatorname{Ln}\left(E T_{t} / L i_{t}\right)$ & $0.208(1.717)$ & \\
\hline \multirow[t]{3}{*}{$\operatorname{Ln}\left[Y_{t} / L_{t}\right]$} & const & $-1.470(3.466)$ & \multirow[t]{3}{*}{0.99} \\
\hline & $\operatorname{Ln}\left(F_{t} / L_{t}\right)$ & 0.149 (1.909) & \\
\hline & $\operatorname{Ln}\left(Y i_{t} / L_{t}\right)$ & $1.131(26.303)$ & \\
\hline
\end{tabular}




\section{СПИСОК ЛИТЕРАТУРЫ}

1. Pearson G., Latanision R.M., Frase K.G. Engineering Technology Education in the United States. National Academies Press, 2016.

2. Reys-Nickel L., Johanna Lasonen J. Graduates of 2-year engineering and engineering technician programmes in the U.S. and the U.K. // Journal of technical education and training. 2018. Vol.10, No.1. DOI: 10.30880/jtet.2018.10.01.003

3. Варшавский A.E. Изменение предпочтений при выборе профессии // Анализ и моделирование экономических и социальных процессов / Математика. Компьютер. Образование: Сб. научн. трудов. Выпуск 25. - М.-Ижевск: НИЦ «Регулярная и хаотическая динамика», 2018. с. 125-139.

4. Комкина T.A. Особенности подготовки кадров в условиях развития цифровой экономики в России // Анализ и моделирование экономических и социальных процессов / Математика. Компьютер. Образование: Сб. научн. трудов. Выпуск 25. - М.-Ижевск: НИЦ «Регулярная и хаотическая динамика», 2018. с. 107-114.

5. Комкина T.A. Анализ подготовки инженерно-технических кадров на основе международных исследований и рейтингов университетов // Анализ и моделирование экономических и социальных процессов / Математика. Компьютер. Образование: Сб. научн. трудов (выпуск 23). № 4. - М.Ижевск: НИЦ «Регулярная и хаотическая динамика», 2016.c. 164-172.

6. Horn L. Stopouts or Stayouts? Undergraduates who leave college in their first year. Washington DC: US Department of Education, 1998, NCES 1999-087.

7. Ryoo J., Rosen S. The Engineering Labor Market// Journal of Political Economy. 2004. Vol. 112, №1. p.110-140.

8. National Center for Education Statistics (NCES). (2017). Percentage of 2011-12 First Time Postsecondary Students Who Had Ever Declared a Major in an Associate's or Bachelor's Degree Program Within 3 Years of Enrollment, by Type of Degree Program and Control of First Institution: 2014. URL: https://nces.ed.gov/datalab/tableslibrary/viewtable.aspx?tableid=11764

9. Roa J.A., Egbert W. What do Educational Mismatches Tell us about Skill Mismatches? A Cross Country Analysis. Paper for the Seminar: European Labour Market of Higher Education Graduates: Analysis of the CHEERS Project Segovia, 2005.

10. Abreu M., Faggian A., McCann Ph. Migration and inter-industry mobility of UK graduates: Effect on earnings and career satisfaction, 51st Congress of the European Regional Science Association: "New Challenges for European Regions and Urban Areas in a Globalised World", 30 August - 3 September 2011, Barcelona, Spain, European Regional Science Association (ERSA), Louvain-la-Neuve, 2011.

11. Half of UK graduates do not work in their field of study, survey reveals. URL: https://www.independent.co.uk/student/news/half-of-uk-graduates-do-notwork-in-their-field-of-study-survey-reveals- $9574042 . \mathrm{html}$ 
12. Robst J. Education and Job Match: The Relatedness of College Major and Work // Economics of Education Review. 2007. №26 (4). p.397-407.

13. Martin N., Persson I., Rooth D.-O. Education-Occupation Mismatch: Is there an Income Penalty? IZA Discussion Paper No.380, 2008.

14. Гимпельсон В.Е., Капелюшников Р.И. и др. Выбор профессии: чему учились и где пригодились // Экономический журнал Высшей школь экономики. 2009. т.13, № 2. с.172-216.

15. Spady, $W$. Dropouts from higher education: An interdisciplinary review and synthesis // Interchange. 1970. Vol. 1. p.64-8.

16. Bean, J.P. Student attrition, intentions, and confidence: Interaction effects in a path model // Research in Higher Education. 1982. Vol.17. p.291-320.

17. Pascarella E., Chapman D. A multi-institutional validation of a theoretical model of college withdrawal // American Educational Research Journal. 1983. Vol.20. p.87-102.

18. Nakosteen R.A, Zimmer M.A. The effects on earnings of interregional and interindustry migration // Journal of Regional Science. 1982. Vol. 22(3). p. 325-341.

19. Stratton L.S. et al. A multinomial logit model of college stopout and dropout behavior // Economics of Education Review. 2008. Vol.27. p.319-331. doi:10.1016/j.econedurev.2007.04.003

20. Bean, J. P. Interaction Effects Based on Class Level in an Explanatory Model of College Student Dropout Syndrome // American Educational Research Journal. 1985. Vol.22, №1. p.55-64.

21. Гуртов В.А., Питухин Е.А., Серова Л.М. Моделирование потребностей экономики в кадрах с профессиональным образованием // Проблемы прогнозирования. 2007. №6. с.91-107.

22. Кочеткова E.B. Моделирование численности выпуска инженернотехнических специалистов (на примере США) // Анализ и моделирование экономических и социальных процессов / Математика. Компьютер. Образование: Сб. научн. трудов (выпуск 23). № 4. - М.-Ижевск: НИЦ «Регулярная и хаотическая динамика», 2016.c. 156-163.

23. Российский статистический ежегодник. Выпуски за 2003-2018 гг.

URL: http://www.gks.ru/wps/wcm/connect/rosstat main/rosstat/ru/statistics/publications/

24. Образование в Российской Федерации: 2014 : статистический сборник. Москва: НИУ «Высшая школа экономики». Выпуски за 2004, 2010, 2014.

25. Вариавский А.Е., Кочеткова Е.В. Моделирование показателей спроса и предложения инженерно-технических специалистов // Экономический анализ: теория и практика. 2018. т. 17, №5. с.886 - 905. doi: 10.24891/ еa. 17. 5.886 


\section{MODELING NUMBER OF ENGINEERING GRADUATES}

\section{Kochetkova E.V.}

The article proposes he model for the number of engineering graduates $d y$ namics providing opportunity to capture the dynamics of enrollment in engineering and other fields as far as modeling the rate of attrition during the education that implies the change in expected return for the future work. 